\title{
Pulling survivors from the wreckage: Resuscitating rejected papers
}

By: Justin Harmon and Kyle M. Woosnam

Harmon, J., \& Woosnam, K.M. (2019). Pulling survivors from the wreckage: Resuscitating rejected papers. Scholé: A Journal of Leisure Studies \& Recreation Education, 34(1), 2935. doi:10.1080/1937156X.2019.1589801

This is an Accepted Manuscript of an article published by Taylor \& Francis in Scholé: A Journal of Leisure Studies \& Recreation Education on 16 May 2019, available online: http://www.tandfonline.com/10.1080/1937156X.2019.1589801.

***(C) 2019 National Recreation and Park Association. Reprinted with permission. No further reproduction is authorized without written permission from Taylor \& Francis. This version of the document is not the version of record. ***

\begin{abstract}
:
$\mathrm{My}^{1}$ initial attempt at a first-authored paper was quite the adventure. I was in the midst of dissertation data collection and my early focus was on the intersection of secular spirituality and music. Someone I had met at a conference forwarded me a CFP for spirituality and music in a religious journal, and I thought it sounded like a good fit. I was working with one of my committee members on the paper, and as she was both the head of the religious studies and performance studies programs, I thought I was set in terms of support. I hadn't really done any thorough vetting of the journal, so when I received an email from them shortly after submission stating that they were interested in publishing it, I was a little wary. Part of this unease came from their declaration that there was a fee of several hundred dollars to do so. While a novice, I still knew something was fishy. I mentioned this to my coauthor and she advised that I withdraw the submission, which I did. Then the editor of the journal said that they liked my paper so much that perhaps they could waive the fee this time. Another red flag. I decided I should take it to one of the journals in the field of leisure studies and try my luck there. So I submitted it to Journal of Leisure Research in August of 2013 where I went through three revisions until it was finally rejected in January of 2015. Despite the progress made, the manuscript was ultimately denied due insufficient progress and inadequate significance. It was a frustrating start to my career. From the first author's journal.
\end{abstract}

Keywords: Rejected papers | revisions | manuscript review | leisure

\section{Article:}

\section{Introduction}

Those in the academy are aware of the "publish or perish" mandate that hangs above their heads. In a recent article in New Republic (July, 2017), David Sessions dissected the demise of the public intellectual, suggesting that with the rise in publication expectations especially in niche areas, coupled with the lessening availability of stable employment in higher education (i.e., 
tenure-track positions), those who are really going to affect the culture of society are forced to find different careers outside of academia, including online blogs, forums, and free presses. For those of us who persist in our calling as academics and scholars and find homes in colleges and universities, we are charged with becoming proficient in conducting research that adds new knowledge to our field. This requires being able to write up the results of our research in such a manner that it not only makes sense and is seen as valuable but resonates with the editor and the review team. While the blind peer-review process is far from perfect, it is the best option currently available (Souder, 2011). The blind peer-review includes experts in the field who are chosen by the journal's editorial staff with the purpose of creating an objective and critical examination of the work in question.

What inevitably comes with the review process, especially for those closer to the beginning of their careers, is a fair dose of rejection. Studies have suggested that the stresses that accompany graduate students and junior faculty in regards to publishing can be quite harrowing, even establishing a connection to anxiety and depression and the publication process (Woolston, 2017). The purpose of this paper is to highlight how I overcame initial frustration and reworked three papers from rejection to publication, and the lessons learned along the way. The question guiding this reflection was, how can I salvage the energy, hard work, and time I put into a rejected paper - all while making a valuable contribution to the field?

\section{Negotiating rejection}

We must accept that scholars at all stages of their career are susceptible to rejection in publishing. While it is not solely junior faculty who have their work dismissed, it is more likely to occur at the beginning of one's career (Altman \& Baruch, 2008). However, rejections should not be viewed entirely as defeats. In fact, rejected papers give the author insights into where they need to focus more attention, how they can add to the literature, as well as their inefficiencies and oversights in the development of their writing style and process (Apel, 2008; Donovan, 2007). Kool, Ziersch, Robinson, Wolfenden and Lowe (2016) suggested there were "seven deadly sins" commonly found in rejected papers: 1) irrelevant topic; 2) lack of focus; 3 ) weak methods; 4) poorly structured results; 5) inadequate analysis; 6) lack of focus on the bigger picture implications for the field; and finally, 7) insufficient writing style.

Dies (1993) stated that many obstacles exist prior to submission that prospective authors have to confront before they are able to write successfully, the first of which involves overcoming the fear of rejection. It is simply a common and necessary part of the learning process of writing well. For Dies, it all starts with the framework. If you have a clear outline of what you want to accomplish and how, then it is easier to pinpoint the areas that are weak and in need of attention, something that you want to do before submission. Klingner, Scanlon, and Pressley (2005) concurred, stating that there must be a clear focus so that the author can write about a specific topic with specific intentions. Klingner et al. go on to say that the paper must come full circle, with the findings connecting to the literature review and the closing discussion connecting to the larger field. These assertions echo several of the "seven deadly sins" of rejected papers (Kool et al., 2016). 
For the uninitiated, these suggestions and warnings may come across as daunting. Apel (2008) suggested that there are parallels between the Olympic athlete's training ritual and the long process of conducting research, writing, submission, revisions, and finally (hopefully) on to publication. And while most who have found their calling in higher education are aware of and understand the expectations associated with publishing, this does not make the hurt or frustration any easier when you have one of your manuscripts rejected. So how do you start over when your paper has been dismissed from a journal?

McGrail, Rickard, and Jones (2006) suggested two places to begin: writing courses and writing support groups. Their research found that not only did writing interventions improve the quality of the product, but this led to an increase in the number of manuscripts published. McRail et al. also found it imperative to refine one's writing style, which includes the interpretation and presentation of data. Donovan (2007) warned against simply taking a "bad" rejected paper and submitting it elsewhere without staging some form of intervention and stressed the importance of adopting the most relevant aspects of the initial reviewers' critique. Altman and Baruch's (2008) results showed that the majority of rejected authors seek out equivalent (if not higher) tier journals to submit to as opposed to lower tier journals, suggesting that many authors take to heart the criticisms and suggestions for improvements given in a thorough review of a rejected paper. All of these suggestions are reactive. Some scholars, including Dies (1993), have suggested that there be a proactive strategy that involves sharing the manuscript with colleagues before submission, potentially inviting them on as coauthors. But even if you choose to get valuable input and feedback from a colleague before submission, this simply does not guarantee your paper will not be rejected. We will now turn our focus to resilience and the resuscitation of the rejected paper to improve it for submission and publication elsewhere.

\section{Iterative process of improving the manuscripts}

To illustrate lessons learned throughout this process, I chose three papers that were rejected from leisure journals, one of which I coauthored with the second author on this effort. All three were eventually revised and reworked and published in other journals in the field of leisure studies. Each of the three manuscripts were rejected for different reasons, and these will be outlined in the results and discussion section. Because of this, the primary methodological examination was textual analysis of the comments and directions from the editors and reviewers of each manuscript. Babbie (2010) stated that textual, or content, analysis involves who says what and why, and to what extent it has an effect on others. In these instances, the "who" were the members of the review team and the "what" were the critiques and guidance of the review team. The "effect" is how the initial feedback was understood and adopted in the revisions postrejection, and what role it played in the submission to another journal.

\section{Data analysis and interpretation}

We relied on Kool et al. (2016) "seven deadly sins" of rejected papers to analyze the missteps in the original submission and eventual rejection of three papers. The analysis process involved the use of a coding technique which began with multiple readings of the editors' and reviewers' comments. Information deemed important based on its relevance to the research question was highlighted. This included the "major" comments from each manuscript provided by the review 
team that were analyzed against Kool et al. "sins" in order to best articulate how the papers were improved for future publication. The "sins," as they fit each essay, became primary codes as part of the initial coding process (Tracy, 2013). Once the categories were created, themes emerged that displayed the context and content of the strategies applied in reworking the manuscripts (Saldaña, 2012). These data provided the foundation of this paper, which reflects on how previously rejected papers were successfully reworked into publishable manuscripts.

\section{Results and discussion}

\section{Paper 1: Clarifying purpose}

(Rejected by Journal of Leisure Research, January 2015, Accepted by Leisure Sciences, September 2015).

As the opening vignette implied, this paper was as much of a learning process as the entirety of my doctoral education (Harmon \& Dox, 2016). While a number of smaller problems nagged the early progress of the manuscript's development, one issue plagued the various iterations, something that took a while to sink in and get right: the purpose of the study. In regards to Kool et al. (2016) "seven deadly sins," while the topic was deemed relevant, the lack of focus (Sin \#2) is what hampered the paper from the beginning. After the first revision, the associate editor (AE) had this to say about the study's purpose:

Clarify your study purpose, and then orient these front sections around it, including your study rationale, theoretical framework, and literature review. In its current form, the manuscript does not offer the reader a thorough enough conceptual review. If you choose to orient the study around spirituality, a lengthy conceptual review will be particularly important as this area of study uses a number of different, and at times conflicting conceptualizations.

Reviewer \#1 added this to the wavering focus of the revision:

The purpose is now stated as a proposition, or almost as a hypothesis, that would be consistent with a positivist study. However, given that this study is an ethnographic, qualitative study I believe the purpose should be written in a much more open ended manner that does not suggest a conclusion prior to the study. For example it could be written as follows: "The purpose of this study is to investigate if and how the passionate fans of the Jackmormons derive spiritual benefit and meaning from their leisure experience of following this band."

I was trying to take on too much in one paper. While there were good ideas presented, I was attempting to blend larger concepts such as secular spirituality and the metaphorical use of "family" (which became its own paper) together, which led to a "watered down" product. In the paper's original conceptualization, I explored not only how the fans addressed their spiritual needs through attendance and engagement at concerts, but also how they formed close friends in the process, many of whom they went on to refer to as "family." What this dual focus did was 
obfuscate the primary purpose (which I was still struggling with): the concept of secular spirituality.

This was pointed out by Reviewer \#1: I was having trouble framing the purpose statement. This is how it initially read: "The purpose of this paper is to posit that passionate fans of the Jackmormons derive spiritual benefit from that involvement." I had already decided that this was the end result. And while this study was embarked upon because I had spoken to many of the fans about their perceived religious and secularly spiritual experiences while participating in the music scene, I had not yet learned why, how, or what earlier experiences they were drawing from. Relying on Reviewer \#1's clarified purpose statement, in addition to reeling in the focus in other areas, allowed for the paper to be a much more streamlined effort.

\section{Paper 2: Defining contribution}

(Rejected by Leisure Sciences, December 2015, Accepted by Annals of Leisure Research, September 2016)

$\mathrm{We}^{2}$ thought we had found a theoretical evolution in a concept we called "leisure expatriation," something we perceived as going beyond the dormant theories of leisure substitutability (Hendee \& Burdge, 1974) and abandonment (Stebbins, 2008). In a paper originally titled, "Leisure expatriation: Building on past experience for future benefit," (Harmon \& Dunlap, 2017) we defined the concept of leisure expatriation as:

The necessity of a pre-existing, high level of immersion in a leisure activity that creates a space for the development of knowledge, skills or interests that ultimately lead to involvement in a similar activity which more fully encapsulates one's leisure goals and desires, and provides justification for abandonment, or the lessening of participation, of a former activity or affiliation.

Try as we might, reviewers for Leisure Sciences did not find that we were drawing any significant differences between the existing concept of substitutability (Hendee \& Burdge, 1974) and our own "novel" idea. For illustration purposes, we had hoped to show that fans with long tenures of participation in a music scene who eventually went on to become more involved in another, but related, music scene could not have done so without the initial exposure and investment in the primary scene. Our declaration was that they had "expatriated" to the new music scene, the way someone might refine their personal interests and life philosophies in their nation of birth and then seek out a country that is more fitting of their desires.

This paper went through three revisions before it was finally dismissed. And similar to the preceding paper on secular spirituality, that is really not as bad as it sounds (Altman \&

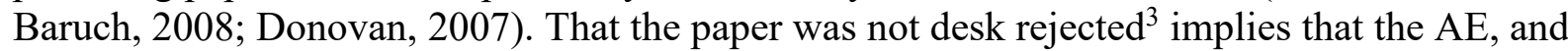
subsequently the reviewers, found merit in the theorizing and writing. However, when considering which element(s) of Kool et al. (2016) "seven deadly sins" apply most, it would have to be Sin \#4: "poorly structured results." Because we could not adequately make the case for a truly new concept, we were perceived to be splitting hairs. Our assertion that evolving out of one leisure pastime into another, even while tangentially related, was neither substantive 
enough in the representation of our findings, nor distinct enough in our delineation from the substitutability concept. The journal failed to see further progress and the paper was rejected.

We put the paper to the side for a short while and then revisited the critiques. After taking some time off and then returning to the paper anew, we felt that after the reviewers pointed out their concerns with the lack of real difference between our concept and Hendee and Burdge's (1974), we should have taken a different strategy: we should have looked at building on the existing concept, not simply trying to move past it. When the paper was rewritten to these specifications, which took very little time, we had truly made a contribution to the literature by showing the evolution of thought from an initial conceptualization over forty years old. If Kool et al. (2016) had an eighth sin, it would have been not seeing the forest for the trees.

\section{Paper 3: Clarity of analysis}

(Rejected by Leisure Studies June, 2016, Accepted By Leisure Sciences, December 2016)

While all rejections are troubling, this one was especially so (Harmon \& Woosnam, 2016). These papers are listed in chronological order of submission, thus showcasing the evolution and maturation of myself in each attempt at publication. While the spirituality and music paper was simply learning the ropes of writing academically as a first author and the substitutability paper was learning to be more practical and developing a better understanding of the groundwork laid by elder scholars, this paper revisited and empirically investigated another older theoretical concept (Clawson \& Knetsch, 1966). Because of the empirical exploration of Clawson and Knetsch's theoretical concept, we found the grounds on which it was dismissed curious. As the title implied ("The temporal phases of leisure experience: Expectation, experience and reflection"), the manuscript explored how the temporal phases of leisure experiences affect future participation decisions, as well as how the continual thread of participation affects the construction of memory. Here is the opening paragraph from the editor's rejection email:

Whilst a really interesting topic and case study, the reviewers point to the need for a more sophisticated theoretical grounding to aid in developing conceptual analysis (and draw out the contribution of the paper) and a location within the events, festival and leisure literatures so as to ensure maximal exposure of the article/relevance to the journal readership. For your information we attach the reviewer comments at the bottom of this email. We hope you will find them to be constructive and helpful. You are of course now free to submit the paper elsewhere should you choose to do so.

The main issue for the review team was our overlooking the tourism literature that was perceived as being imperative to the study. Reviewer \#1 said this: "The ever growing body of literature on events and festivals is ignored altogether, and the broader context within which the findings and implications are discussed, is therefore not convincing." Yet, the paper was not about tourism. While the study took place around a small music festival, the emphasis was on the exploration of how expectations affected participation and memory - not the explicit aspect of being in a small music festival. We were not attempting to explore marketing or any aspect of customer hospitality, economic impact, or engagement with local culture. In fact, one of the findings was that consumer need to be given more control of their leisure experience and the orchestrators of 
events less. The review team for this paper viewed our deductions, and therefore the implications, to be inadequate, reflecting Kool et al.’s (2016) Fifth Sin.

However, the reality was that, as sometimes happens, you do not always draw the most appropriate pool of reviewers for the review of your paper. But before you gasp and brand me with a scarlet letter I should emphasize that this was our fault, not the fault of the AE or review team. We failed to emphasize our explicit focus (Sin Two) thus leaving the interpretation to the AE. Therefore, when we decided to submit to Leisure Sciences, we told the editor about our issue, and asked how we might resolve it before our re-submission. The editor indicated that we should, one, clearly state our emphasis upfront, and two, address some of the tourism literature that does speak to our topic, even if only tangentially. With this guidance we got the paper up to the standards of Leisure Sciences in no time, and after a minor revision, the AE left us with this parting comment:

Congratulations on a set of excellent revisions to this manuscript. All reviewers agree that this paper is ready to be fully accepted for publication. In particular, I think that the new opening is really excellent; we all appreciated the clarity and thoroughness with which you made changes throughout. I'll also echo the 2nd reviewer, who complemented the new, combined Results and Discussion section - very nicely done. I look forward to seeing this one in print, and for use in the classroom. Bravo.

\section{Conclusion}

The takeaway is that the academic writing and publishing process is a constant learning experience. That we are always continually building on existing science, and an established literature base, requires us to not only be well-read in our field and beyond, but that we must have a clear understanding of how what it is we are doing fits, and matters, to the bigger picture of scholarship. While nearly one-third of authors will not modify their paper before submitting it elsewhere (Altman \& Baruch, 2008), we feel that it is always best to take into consideration the comments and critiques of the review team so as to avoid having to confront those headaches upon submission elsewhere. In each instance reported here, rejection was frustrating, albeit for different reasons. However, for me, these rejections were imperative to academic growth (Donovan, 2007). Had I stuck with the initial journal for the music and spirituality paper (the one before $J L R$, the "pay for play" outlet), I would not have grown as an author or investigator. In fact, I may have sought out lower tiered journals that are easier in which to publish, potentially never really making any contribution to a broader readership. And while none of my publications to date have attained "superstar" status in any way, shape, or form, I have learned to see where I can make small contributions which may lead to bigger ones down the road.

Good social science articles test theories, and therefore it all starts with the initial problem statement and research question, and a clear focus for where you are headed (Klingner et al., 2005). But the review process should be expected to be rigorous, and the revision process to be potentially elaborate and time-consuming in most cases. While some scholars recommend the use of writing teams, writing courses, and writing support groups before submission (McGrail et al., 2006), it may be best to look at the AE and reviewers of your manuscript as those resources after submission - see them as collaborators in some way. It is easy to take a 
review as overly harsh, even cynical, but it is nothing more than a guide - and a challenge - for you to advance the field in some way.

Now that I have started to understand what is valuable to the field of leisure studies through my own scholarship, I have been given the opportunity to serve as both an associate editor and reviewer numerous times, tasks I do not take lightly. While I approach each submission with a critical eye, I always try to leave the author(s) with a clear path forward for improving their manuscript. It is our job as editors and reviewers to help shepherd emerging scholars into the field, and if we solely rely on a biting tongue and wagging finger in our reviews, then we are doing a disservice to the field. This does not mean we should not be direct and challenging - by all means we need to be. But it does mean that we have to be conscious of how our recommendations and tone will affect the author and his/her development as a scholar and contributor to the field. Fortunately I am not too far removed from my most demanding (and seemingly impossible to overcome) reviews to know how welcoming a wise and helping hand can be.

Kool et al. (2016) cited the Scottish philosopher Thomas Carlyle in the final paragraph of their piece, and we think it bears repeating: "Permanence, perseverance, and persistence in spite of all obstacles, discouragements and impossibilities." Making contributions to science and society is not easy, and not always straight-forward. But we should always strive to retain our innerstudent, always be willing to take the advice of those who are there to give it. Frustration should lead to motivation, and motivation, steadfastly applied, should lead to publication and scholarly growth. ${ }^{4}$

\section{Notes}

1. The pronouns "my" and "I" refer to the first author throughout.

2. "We" refers to the first and second authors of this manuscript.

3. To "desk reject" a paper would be for the Editor to reject it immediately upon submission without sending out to an associate editor for an initial review.

4. For a good resource in getting started, check out the work by White (2005).

\section{References}

Altman, Y., \& Baruch, Y. (2008). Strategies for revising and resubmitting papers to refereed journals. British Journal of Management, 19(1), 89-101.

Apel, K. (2008). The publication process: An Olympic feat. Language, Speech, and Hearing Services in Schools, 39(4), 439. doi:10.1044/0161-1461(2008/ed-04)

Babbie, E. R. (2010). The practice of social research. Stamford, CT: Cengage Learning.

Clawson, M., \& Knetsch, J. L. (1966). Economics of outdoor recreation. Baltimore, MD: The John Hopkins Press. doi:10.1086/ahr/52.4.744

Dies, R. R. (1993). Writing for publication: Overcoming common obstacles. International Journal of Group Psychotherapy, 43(2), 243-249. doi:10.1080/00207284.1994.11491220 
Donovan, S. K. (2007). The importance of resubmitting rejected papers. Journal of Scholarly Publishing, 38(3), 152-157. doi:10.3138/w706-5884-kg84-j282

Harmon, J., \& Dox, D. (2016). Spirituality, fan culture, and the music of Jerry Joseph \& the Jackmormons. Leisure Science, 38(3), 268-283. doi:10.1080/01490400.2015.1092404

Harmon, J., \& Dunlap, R. (2017). The temporal phases of leisure experience: Expectation, experience and reflection of leisure participation. Leisure Sciences, 40(5), 326-342. doi:10.1080/01490400.2016.1274246

Harmon, J., \& Woosnam, K. M. (2016). Extending the leisure substitutability concept. Annals of Leisure Research, 21(4), 424-439. doi:10.1080/11745398.2016.1235485

Hendee, J. C., \& Burdge, R. W. (1974). The substitutability concept: Implications for recreation research and management. Journal of Leisure Research, 6(2), 157-162.

Klingner, J. K., Scanlon, D., \& Pressley, M. (2005). How to publish in scholarly journals. Educational Researcher, 34(8), 14-19. doi:10.3102/0013189x034008014

Kool, B., Ziersch, A., Robinson, P., Wolfenden, L., \& Lowe, J. B. (2016). The 'seven deadly sins' of rejected papers. Australian and New Zealand Journal of Public Health, 40(1), 3-4.

McGrail, M. R., Rickard, C. M., \& Jones, R. (2006). Publish or perish: A systematic review of interventions to increase academic publication rates. Higher Education Research \& Development, 25(1), 19-35. doi:10.1080/07294360500453053

Saldaña, J. (2012). The coding manual for qualitative researchers. Thousand Oaks, CA: Sage.

Sessions, D. (2017, July). The rise of the thought leader. New Republic, 48-52.

Souder, L. (2011). The ethics of scholarly peer review: A review of the literature. Learned Publishing, 24(1), 55-74.

Stebbins, R. A. (2008). Leisure abandonment: Quitting free-time activity that we love. LSA Newsletter 81. Leisure Reflections, 19, 14-19.

Tracy, S. (2013). Qualitative research methods: Collecting evidence, crafting analysis, communicating impact. Hoboken, NJ: Wiley-Blackwell.

White, L. (2005). Writes of passage: Writing an empirical journal article. Journal of Marriage and Family, 67(4), 791-798. doi:10.1111/j.1741-3737.2005.00175.x

Woolston, C. (2017). Graduate survey: A love-hurt relationship. Nature, 550(7677), 549-552. doi:10.1038/nj7677-549a 